\title{
Activation of epithelial CD98 glycoprotein perpetuates colonic inflammation
}

\author{
Torsten Kucharzik ${ }^{1}$, Andreas Lugering ${ }^{1}$, Yutao Yan ${ }^{2}$, Adel Driss ${ }^{2}$, Laetitia Charrier $^{2}$, \\ Shanthi Sitaraman ${ }^{2}$ and Didier Merlin ${ }^{2}$ \\ ${ }^{1}$ Department of Medicine B, Münster University of Münster, Germany and ${ }^{2}$ Department of Medicine, \\ Division of Digestive Diseases, Emory University School of Medicine, Atlanta, GA, USA
}

\begin{abstract}
Anomalies in the regulation and function of integrins have been implicated in the etiology of various pathologic conditions, including inflammatory disorders such as irritable bowel disease. Several classes of cell surface glycoproteins such as CD98 have been shown to play roles in integrins-mediated events. Here, we investigated the role of CD98 in intestinal inflammation using both in vivo and in vitro approaches. We found that in Caco2BBE monolayers and colonic tissues, expression of CD98 was upregulated by the proinflammatory cytokine, interferon gamma (INF $\gamma$ ). Furthermore, CD98 was highly upregulated in colonic tissues from mice with active colitis induced by dextran sodium sulfate (DSS), but not in DSS-treated INF $\gamma-I$ - mice. Administration of an anti-CD98 antibody worsened DSS-induced colitis in mice but had no effect on untreated control mice. Finally, we used Caco2-BBE cell monolayers to model intestinal epithelial wound healing, and found that activation of epithelial CD98 in DSS-treated monolayers inhibited monolayer reconstitution, but had no affect on untreated control monolayers. Our data collectively indicate that (i) CD98 upregulation is mediated by INF $\gamma$ during intestinal inflammation and (ii) activation of epithelial CD98 protein aggravates intestinal inflammation by reducing intestinal epithelial reconstitution. Overall, our data suggest that epithelial CD98 plays an important role in the perpetuation of intestinal inflammation.
\end{abstract}

Laboratory Investigation (2005) 85, 932-941. doi:10.1038/labinvest.3700289; Published online 9 May 2005

Keywords: CD98; DSS model; Caco2-BBE monolayers

The regulation and establishment of the major epithelial permeability barrier is the end result of a cascade of events triggered by cell-cell and cellextracellular matrix (ECM) interactions. Anomalies in the regulation and function of integrins have been implicated in the etiology of various pathologic conditions, including inflammatory disorders such as inflammatory bowel disease (IBD). ${ }^{1-3}$

Several classes of cell surface glycoproteins have been shown to play a role in integrins-mediated events, including CD98, CD36, CD63, CD9. Of these, CD98 is a cell surface heterodimer formed by covalent linkage of a $\sim 80 \mathrm{kDa}$ heavy chain (hc) and $\sim 40 \mathrm{kDa}$ light chain (lc). ${ }^{4,5}$ CD98 was initially characterized as a T-cell activation marker, and was later shown to function as an amino-acid transporter. $^{6,7}$ Recent work demonstrated that a CD98/LAT-2 heterodimer is found in tissues containing epithelial

Correspondence: Dr D Merlin, PhD, Department of Medicine, Division of Digestive Diseases, Emory University, 615 Michael Street, Atlanta, GA 30322, USA.

E-mail: dmerlin@emory.edu

Received 1 February 2005; revised 30 March 2005; accepted 31 March 2005; published online 9 May 2005 barriers. ${ }^{8-10}$ We previously demonstrated that $\beta 1$ integrins, which are also basolaterally polarized in intestinal epithelial cells, associate with CD98/LAT-2, and that CD98 influences $\beta 1$ integrins distribution as well as cell shape and cytoskeletal organization, which depend on the function of $\beta 1$ integrins. $^{9}$ Interestingly, CD98 interacts specifically with $\beta 1$ integrins, but not with the muscle-specific splice variant $\beta 1 \mathrm{D}$, or the leukocyte-specific $\beta 7$ integrins. ${ }^{11}$ The basolateral location of CD98 suggests that this protein could be involved in some form(s) of cell signaling where binding of a ligand to the extracellular loop of CD98 results in cellular changes through regulation of an amino-acid transporter (LAT-2 in the intestine) and the function of $\beta 1$ integrins. $^{9,10}$ We previously demonstrated that ICAM-1 co-precipitates with the CD98/LAT2 heterodimer in intestinal epithelial cells, suggesting that ICAM-1 functions not only as an individual receptor but also as a component of supramolecular complexes at the plasma membrane in epithelial cells. ${ }^{12}$

In the context of intestinal inflammation, CD98 protein expression in human colonic epithelium was shown to be upregulated by proinflammatory cytokines such as Interferon $\gamma$ (INF $\gamma),{ }^{13}$ and 
increased expression of lymphocyte activation antigens for CD98 was found on the cell surface of intestinal B cells, T cells, CD4 ${ }^{+} \mathrm{T}^{-}$cells, and $\mathrm{CD}^{+}{ }^{+} \mathrm{T}$ cells isolated from patients with Crohn's disease and ulcerative colitis. ${ }^{14}$ These and other studies have revealed that markedly increased intestinal lymphocyte activation is a major immunological alteration in IBD. ${ }^{15}$ In addition, 5-aminosalicylic acid (which is used for the treatment of intestinal inflammation in IBD) dose-dependently inhibited the expression of the CD98 cell-surface activation antigen on mitogen-activated peripheral blood lymphocytes, further suggesting that CD98 plays an important inflammatory role. ${ }^{14}$ However, although these previous studies have clearly shown that CD98 likely plays an important role in intestinal inflammation, no previous work has studied the role of epithelial CD98 in intestinal inflammation. Here, we used a combination of in vivo and in vitro approaches to investigate the role of epithelial CD98 in intestinal inflammation.

\section{Materials and methods}

\section{Cell Culture and Treatments}

Caco2-BBE ${ }^{16-19}$ cells (between passages 30 and 50) were grown in high glucose Dulbecco's Vogt Modified Eagle's Media (DMEM; Invitrogen, Carlsbad, CA, USA) supplemented with $14 \mathrm{mmol} / \mathrm{l} \mathrm{NaHCO}_{3}$, and $10 \%$ newborn calf serum. Cells were incubated at $37^{\circ} \mathrm{C}$ in $5 \% \mathrm{CO}_{2}$ and $90 \%$ humidity, and the medium was changed daily. Monolayers were subcultured every 7 days with $0.1 \%$ trypsin and $0.9 \mathrm{mmol} / \mathrm{l}$ ethylenediaminetetraacetic acid (EDTA) in $\mathrm{Ca}^{2+} / \mathrm{Mg}^{2+}$-free phosphate-buffered saline (PBS). Experiments were performed with confluent monolayers plated on permeable supports (area $1 \mathrm{~cm}^{2}$; pore size $0.4 \mu \mathrm{m}$; Transwell-Clear polyester membranes; Costar (Life Sciences, Acton, MA, USA) and examined 15 days postplating. IFN $\gamma(100 \mathrm{U} / \mathrm{ml}$ in serum-free medium) was added to the basolateral aspect of the monolayers and incubated for $48 \mathrm{~h}$. Recombinant IFN $\gamma$ (produced in Escherichia coli and having a specific activity of $2 \times 10$ (REF) U/mg) was obtained from Genentech (San Francisco, CA, USA).

\section{Western Blot Analysis}

Colonic mucosal biopsies were collected from patients who underwent colonoscopy for polyp or cancer surveillance. All subjects were given informed consent, as approved by Human Investigations Committee of Emory University Atlanta, GA. Human colonic mucosa biopsies and mouse colonic mucosa (the muscle layer was carefully removed using pointed forceps) were lysed with PBS containing $1 \%$ Triton X-100 and $1 \%$ NP-40 (v/v), protease inhibitor cocktail (Boehringer Mannheim), EDTA, SDS, sodium orthovanadate, and sodium fluoride.

Proteins were separated by SDS-polyacrylamide gel electrophoresis on $4-20 \%$ gradient gels (BioRad, Hercules, CA, USA), and then transferred to nitrocellulose membranes. The blots were blocked for $1 \mathrm{~h}$ with 5\% nonfat milk in blocking buffer, and then incubated for $1 \mathrm{~h}$ with $1 / 100$ dilutions of goat polyclonal anti-human CD98 (Santa Cruz Biotechnology, Santa Cruz, CA, USA), goat polyclonal antimouse CD98 (Santa Cruz) or goat polyclonal anti-ERK (Santa Cruz). After the blots were washed three times for $15 \mathrm{~min}$ in blocking buffer, they were further incubated for $1 \mathrm{~h}$ with the corresponding secondary horseradish peroxidase (HPR) conjugated anti-goat antibody (diluted 1:2000). The membranes were then washed three times (20 min each) in blocking buffer and then probed using a chemiluminescence system (ECL; Amersham, Piscataway, NJ, USA).

\section{Membrane Extractions}

Caco2-BBE pellets were resuspended and homogenized in HEPES ( $5 \mathrm{mM}$ ) containing protease inhibitors. The samples were then incubated for $30 \mathrm{~min}$ at $4^{\circ} \mathrm{C}$ and centrifuged at $13000 \mathrm{~g}$ for $30 \mathrm{~min}$ at $4^{\circ} \mathrm{C}$. The resulting pellet was suspended in PBS by repeated passage through an 18-gauge needle. The protein solution was boiled for $5 \mathrm{~min}$ at $100^{\circ} \mathrm{C}$ in Laemmli buffer supplemented with $0.5 \% \beta$-mercaptoethanol and then used for protein separation on SDS-polyacrylamide gel electrophoresis.

\section{Confocal Immunofluorescence}

Caco2-BBE cells grown on filters were washed twice in Hank's balanced salt solution (HBSS), $\mathrm{pH} 7.4$, and then fixed with $3.7 \%$ paraformaldehyde in HBSS with calcium, pH $7.4\left(\mathrm{HBSS}^{+}\right)$. The cells were then permeabilized with $0.5 \%$ Triton for $30 \mathrm{~min}$ at $25^{\circ} \mathrm{C}$, rinsed and incubated with rhodamine-phalloidin (Molecular Probes, Eugene, OR, USA) diluted 1:60 for $40 \mathrm{~min}$. The samples were then blocked for $1 \mathrm{~h}$ in a blocking solution containing $0.2 \%$ gelatin and $0.08 \%$ saponin in $\mathrm{HBSS}^{+}$and incubated for $1 \mathrm{~h}$ with $2 \mu \mathrm{g} / \mathrm{ml}$ goat anti-human CD98 antibody (Santa Cruz, CA, USA). The monolayers were stained with the appropriate fluorescein isothiocyanate antibody diluted 1:1000 (Molecular Probes, OR, USA). Microscopy was performed with a Zeiss epifluorescence microscope equipped with a BioRad MRC600 confocal unit, computer and laser scanning microscope (LSM) image analysis software (Zeiss, Jena, Germany).

\section{Animals}

All studies were carried out in female C57BL/6 mice (8 weeks, 18-22 g) or female C57BL/6 IFN $\gamma^{-1-}$ mice 
(8 weeks, 18-22g) obtained from Jackson Laboratories (Bar Harbor, ME, USA). Mice were grouphoused under a controlled temperature $\left(25^{\circ} \mathrm{C}\right)$ and photoperiod (12:12-h light-dark cycle). The mice were allowed unrestricted access to standard mouse chow and tap water. They were allowed to acclimate to these conditions for at least 7 days before inclusion in experiments. The animals were treated in accordance with the Emory University Institutional Animal Care, Authorization no. 146-2002.

\section{Induction of Colitis and Study Design}

Colitis was induced in all groups by the addition of $3 \%(\mathrm{w} / \mathrm{v})$ dextran sodium sulfate (DSS; molecular weight $40 \mathrm{kDa}$; (ICN Biochemicals, Auora, OH, USA) to the drinking water. Mean DSS/water consumption and body weight were assessed daily during the treatment period. Six groups of mice $(n=6$ per group) were treated for 7 days with DSS alone, DSS plus daily intraperitoneal (i.p.) injections of $0.5 \mathrm{mg} /$ ml CD98 polyclonal goat anti-mouse antibody (Santa Cruz), DSS plus $0.5 \mathrm{mg} / \mathrm{ml}$ isotype control (daily by i.p.), NDW plus $0.5 \mathrm{mg} / \mathrm{kg}$ anti-CD98 (daily by i.p.), or normal drinking water plus $0.5 \mathrm{mg} / \mathrm{ml}$ isotype control (daily by i.p.).

\section{IFN $\gamma$ Treatment in Mice}

Recombinant rIFN $\gamma$ (specific activity of $2 \times 10$ (REF) U/mg units/mg; Genentec) was administrated i.p. at a concentration of $10^{5}$ units/day for 3 days.

\section{Histology}

To assess the distribution of colonic inflammatory changes, colons were dissected from mice, cut into three equal sections and attached to a cork board before fixation with $2 \%(\mathrm{v} / \mathrm{v})$ paraformaldehyde solution. Samples were embedded in paraffin and sectioned. Hematoxylin and eosin (H\&E) staining was used for general assessment of intestinal inflammation, which was performed by a GI pathologist blinded to the treatment protocol. Each section was scored for ulceration severity and extent, and the tissue thickness from the muscularis propria to the luminal border was determined. Lesion severity was graded using a modification of a previously defined scoring system with a scale of 0-4: 0 normal; 1 minimal; 2 mild; 3 moderate; 4 severe..$^{20}$ Minimal lesions contained small, focal, or widely dispersed areas of inflammation and/or fibrosis above the muscularis mucosae. Mild lesions were multifocal or locally extensive and contained inflammation or fibrosis extending into the submucosa. Moderate lesions consisted of multifocal lesions with ulcers consuming less than $10 \%$ of the assessed mucosal surface. Severity was defined as having moderate lesions and/or ulcers consuming more than $10 \%$ of the assessed mucosal surface.

\section{Wound-Healing Assays}

Wound-healing assays were performed with the Electric Cell-Substrate Impedance Sensing (ECIS Model 1600R (Applied BioPhysics, Inc., Troy, NY, USA) technology. The ECIS device is based on AC impedance measurements using weak and noninvasive AC signals, as previously described. ${ }^{21,22}$ Attachment and spreading of cells on the electrode surface changes the impedance, allowing inference of morphological information. The measurement system consists of an eight-well culture dish with electrodes $\left(0.0005 \mathrm{~cm}^{2}\right)$ at the bottom of each well, a lock-in amplifier with an internal oscillator, relays to switch between the different wells, and a personal computer that controls the measurement and stores the data. The entire system was obtained from Applied Biophysics, Inc.

We first determined the ideal frequency for measuring the resistance of confluent Caco2-BBE monolayers by taking resistance measurements during a frequency scan of cells plated on ECIS 8W1E plates (Applied BioPhysics). The cells were then removed by trypsinization, and the resistance measurement was repeated on the naked electrode. The ideal frequency was that at which the ratio of the Log resistance with cells over the Log resistance without cells reached its peak. For Caco2-BBE cells, we determined the ideal frequency to be $500 \mathrm{~Hz}$ at a voltage of $1 \mathrm{~V}^{23}$

For experiments, we first flooded the ECIS $8 \mathrm{~W} 1 \mathrm{E}$ electrode with a solution of $10 \mu \mathrm{g} / \mathrm{ml}$ goat antimouse CD98 antibody or $10 \mu \mathrm{g} / \mathrm{ml}$ goat isotype control for $60 \mathrm{~min}$, and then washed the electrode with serum-free medium to remove nonadherent proteins. Caco2-BBE cells were then plated at $0.2 \times 10^{6}$ cells per well; at confluency, the monolayers were treated with $3 \%$ DSS or vehicle and incubated for a further $20 \mathrm{~h}$. The cells were then exposed to an elevated voltage pulse $(40 \mathrm{kHz}$ and $4.5 \mathrm{~V}$ for $30 \mathrm{~s}$ ) for wounding, and resistance measurements were sampled from each well every $30 \mathrm{~s}$ continuously for about $20 \mathrm{~h}$, as previously described. ${ }^{23,24}$

\section{Statistical Analysis}

The differences between mouse body weights, bowel lengths, and number of intestinal ulcers were compared using Mann-Whitney $U$ statistics supported by the InStat software for Macintosh computers.

\section{Results}

\section{Interferon $\gamma$ Increases CD98 Protein Expression in Caco2-BBE Monolayers}

To investigate the effect of IFN $\gamma$ on CD98 expression, we incubated Caco2-BBE monolayers for $48 \mathrm{~h}$ in serum-free medium with $100 \mathrm{UI} / \mathrm{ml} \mathrm{IFN} \gamma$ added to 
the basolateral compartment. Previous work showed that the cell polarity is not affected under these treatment conditions. ${ }^{19}$ At the end of the incubation period, we investigated CD98 protein expression using confocal microscopy and Western blotting. Confocal microscopy of confluent Caco2-BBE cell monolayers revealed that the staining for immunoreactive CD98 in control cells was localized to the basolateral domain, mainly in the tight junction area (Figure 1a). After stimulation with IFN $\gamma(100 \mathrm{UI} / \mathrm{ml}$ for $48 \mathrm{~h}$ ), CD98 staining was increased and was additionally found intracellularly and in the subapical membranes (Figure 1b). To confirm these observations, Caco2-BBE cell membranes were isolated from Caco2-BBE cells treated with or without IFN $\gamma$ for $48 \mathrm{~h}$ and Western blotting was performed on membrane and cytosolic fractions, using a goat anti-human CD98 antibody. As previously reported, Caco2-BBE cell lysates displayed a single immunoreactive band corresponding to $\sim 90 \mathrm{kDa}$ in the presence of $\beta$-mercaptoethanol. As shown in Figure 1c, treatment with IFN $\gamma(100 \mathrm{U} / \mathrm{ml}$ for $48 \mathrm{~h})$ increased the membrane CD98 expression in Caco2-BBE cells in comparison to untreated Caco2BBE monolayers (Figure 1c, lane 2 vs lane 4). Together, these results demonstrate that the cytokine, IFN $\gamma$, increases membrane expression of the CD98 protein. Consistent with our confocal results, CD98 was not detectable in the cytosol of untreated Caco2-BBE cells (Figure 1c, lane 1), but was observed in the cytosol of Caco2-BBE cells treated with IFN $\gamma(100 \mathrm{U} / \mathrm{ml})$ for $48 \mathrm{~h}$ (Figure 1c, lane 3). These results suggest that IFN $\gamma$ is likely to increase CD98 expression at the transcriptional level.

\section{CD98 is Expressed In mouse Colonic Tissue}

We then examined CD98 expression in the mouse intestine. The human and mouse CD98 proteins are

a
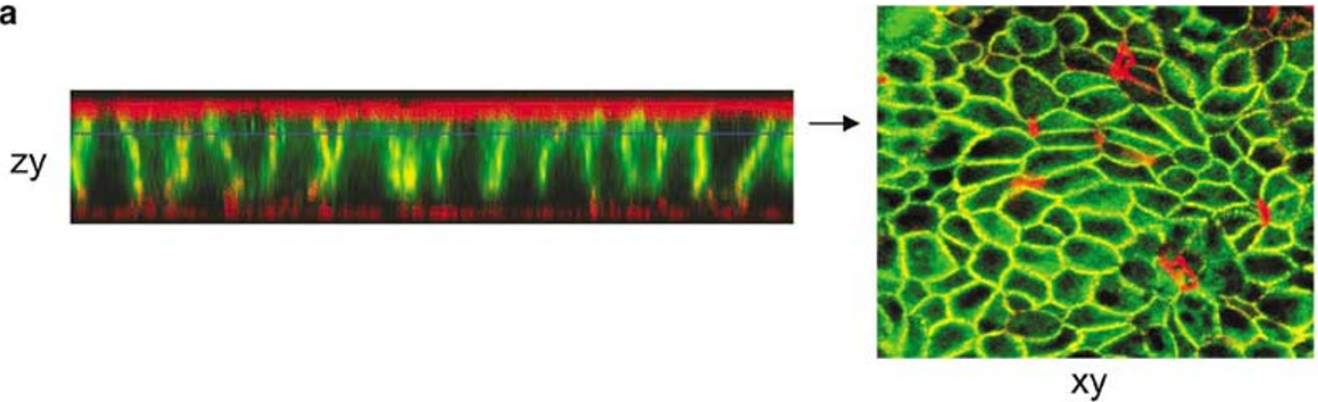

Control

b
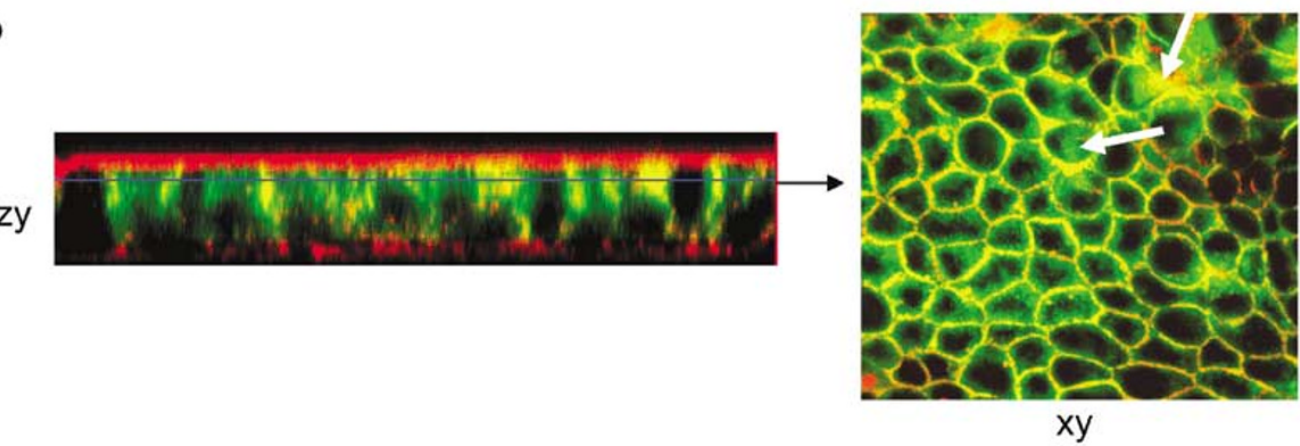

\section{+ Interferon $\gamma$ (100Ul/ml for $48 \mathrm{hrs})$}

C

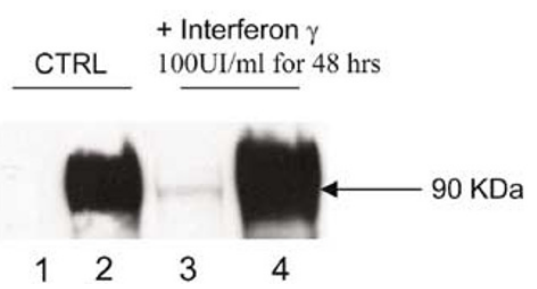

Figure 1 IFN $\gamma$ increases CD98 protein expression in Caco2-BBE monolayers. Confocal microscopic localization of CD98 (green) in Caco2-BBE cell monolayers costained with rhodamine phalloidin (actin, red). Horizontal (xy) below the apical plasma membrane and vertical (zy) sections of polarized Caco2-BBE monolayers were stained with human goat anti-CD98 antibody and also stained with rhodamine-phalloidin. Caco2-BBE cell monolayers were untreated (a) or treated with $100 \mathrm{UI} / \mathrm{ml} \mathrm{IFN} \gamma$ for $48 \mathrm{~h}$ (b). Arrows depict the induction of intracellular CD98 protein by IFN $\gamma$. (c) Caco2-BBE cell monolayers were preincubated with $1000 \mathrm{UI} / \mathrm{ml}$ IFN $\gamma$ for $48 \mathrm{~h}$ and subject to membrane and intracellular protein extraction. Intracellular proteins ( $50 \mu \mathrm{g}$; lanes 1 and 3 ) and $50 \mu \mathrm{g}$ membrane proteins (lanes 2 and 4) from Caco2-BBE cells left untreated (CTRL) or treated with $1000 \mathrm{UI} / \mathrm{ml}$ IFN $\gamma$ for $48 \mathrm{~h}$ were subjected to $4-20 \%$ SDSpolyacrylamide gel electrophoresis (reducing conditions), followed by transfer to a nitrocellulose membrane. The blot was immunostained with a human goat anti-CD98 antibody. The results presented are representative of six different experiments. 
$85 \%$ homologous at the amino-acid level. The antihuman CD98 antibody utilized in this work was raised against a peptide corresponding to the carboxy terminus of human CD98 (amino acids 105-529). We found that this antibody did not crossreact with anti-mouse CD98. In contrast, the utilized anti-mouse CD98 monoclonal antibody (Research Diagnostics) reacted to CD98 in the mouse large intestine (Figure 2a, lane 3), as well as to human CD98 from Caco2-BBE cells and human colon tissue (Figure 2a, lanes 1 and 2). These results suggest that the anti-mouse CD98 recognizes an antigen that is present in both human and mouse CD98 proteins.

\section{Interferon $\gamma$ Upregulates CD98 Expression in Mice Colonic Tissues}

Based on our in vitro results showing that IFN $\gamma$ increases CD98 expression in Caco2-BBE monolayers, we investigated the effect of IFN $\gamma$ in mouse colonic tissues. As shown in Figure 2b, when mice were treated with $10^{5}$ units of IFN $\gamma$, the expression of CD98 in colonic tissues increased by 1 day post-treatment and was sustained through day 3 post-treatment.

\section{Anti-CD98 Does Not Induce Colonic Inflammation in Mice}

Previous studies have reported that monoclonal anti-mouse CD98 activates intracellular signaling events in mouse lymphocytes, ${ }^{24}$ so we examined the effect an anti-mouse CD98 monoclonal antibody on intestinal inflammation in mice. The monoclonal anti-mouse CD98 antibody $(2 \mathrm{mg} / \mathrm{kg})$ was injected i.p. into mice daily for 7 days. As shown in Figure $3 \mathrm{~b}$, there was no macroscopic evidence of inflammation in any part of the colon at day 7 when compared to colons of untreated animals (Figure 3a and Histological activity score: 0). In addition, administration of the anti-CD98 antibody did not induce weight loss during the 7-day treatment period (Figure 4a).

\section{The Course of DSS-Induced Murine Colitis is Aggravated by CD98 Activation}

C57BL/6 mice were exposed to 3\% DSS for a 7-day period, resulting in intestinal colitis (Figure 3c). Histological studies showed that administration of DSS induced cellular damage with epithelial exfoliation, which was not observed in control animals (Figure 3a). We observed interstitial edema, dilation of vessels, and a general increase in inflammatory cells (lymphocytes and polynuclear cells) in the lamina propria of DSS-treated animals (Figure 3c and Histological activity score: 3 ). In addition, the
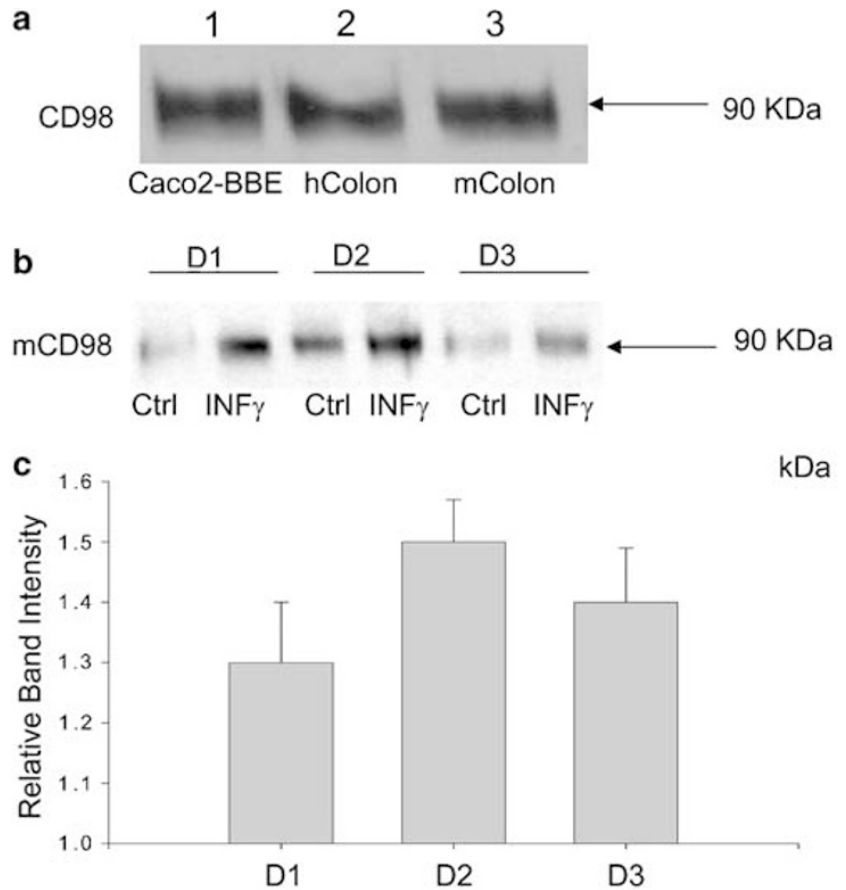

Figure 2 CD98 is expressed in human and mouse large intestine. (a) Western blot analysis of total cell lysates (reducing conditions) from Caco2-BBE cells (Caco2-BBE), human colonic tissue (hColon), and mice colonic tissue (mColon). Total cell proteins (50 $\mu \mathrm{g} /$ lane) were subjected to $7.5 \%$ SDS-polyacrylamide gel electrophoresis followed by transfer to a nitrocellulose membrane. The blot was immunostained with a goat anti-mouse CD98 antibody. (b) Western blot analysis of CD98 protein in mouse intestines after exposure to IFN $\gamma$. Adult mice were injected i.p. (once every day) with $10^{5}$ units of murine recombinant IFN $\gamma$ and killed at days 1 (D1), 2 (D2), and 3 (D3). Colonic segments were harvested for measurement of CD98 protein content. Total proteins $(50 \mu \mathrm{g})$ were subjected to $7.5 \%$ SDS-polyacrylamide-gel electrophoresis followed by transfer to nitrocellulose membrane. The blot was immunostained with a goat anti-mouse CD98 antibody, and then the blot was stripped and reprobed with anti-ERK to demonstrate equivalent loading. Ctrl, untreated; INF $\gamma$, IFN $\gamma$-treated. The results presented are representative of six different experiments. (c) Western blots were quantified using Fluorchem (camera imaging densitometer, model 8300; Alpha Innotech Corp, San Leandro, CA, USA). Protein bands in the autoradiograms were quantified by scanning densitometry and histograms of the densities relative to vehicle-treated controls. Each bar represents the average \pm s.e. of three different autoradiograms.

DSS-treated mice showed weight loss beginning at day 4 and peaking at day 7 (Figure 4a).

To evaluate the role of CD98 in an inflammatory context, anti-mouse CD98 was injected i.p. at $0.5 \mathrm{mg} / \mathrm{kg}$ every day in combination with the administration of DSS until mice were killed at day 7. As shown in Figure 3d, treatment with the anti-mouse CD98 increased the severity of DSS-induced colitis. Histological studies revealed complete erosion of the epithelium, longer ulcerations, and massive crypt loss and marked lamina propria and submucosa mixed inflammatory cell infiltration (Histological activity score: 4). In addition, administration of the anti-CD98 antibody in DSS-induced colitis increased the severity of weight 

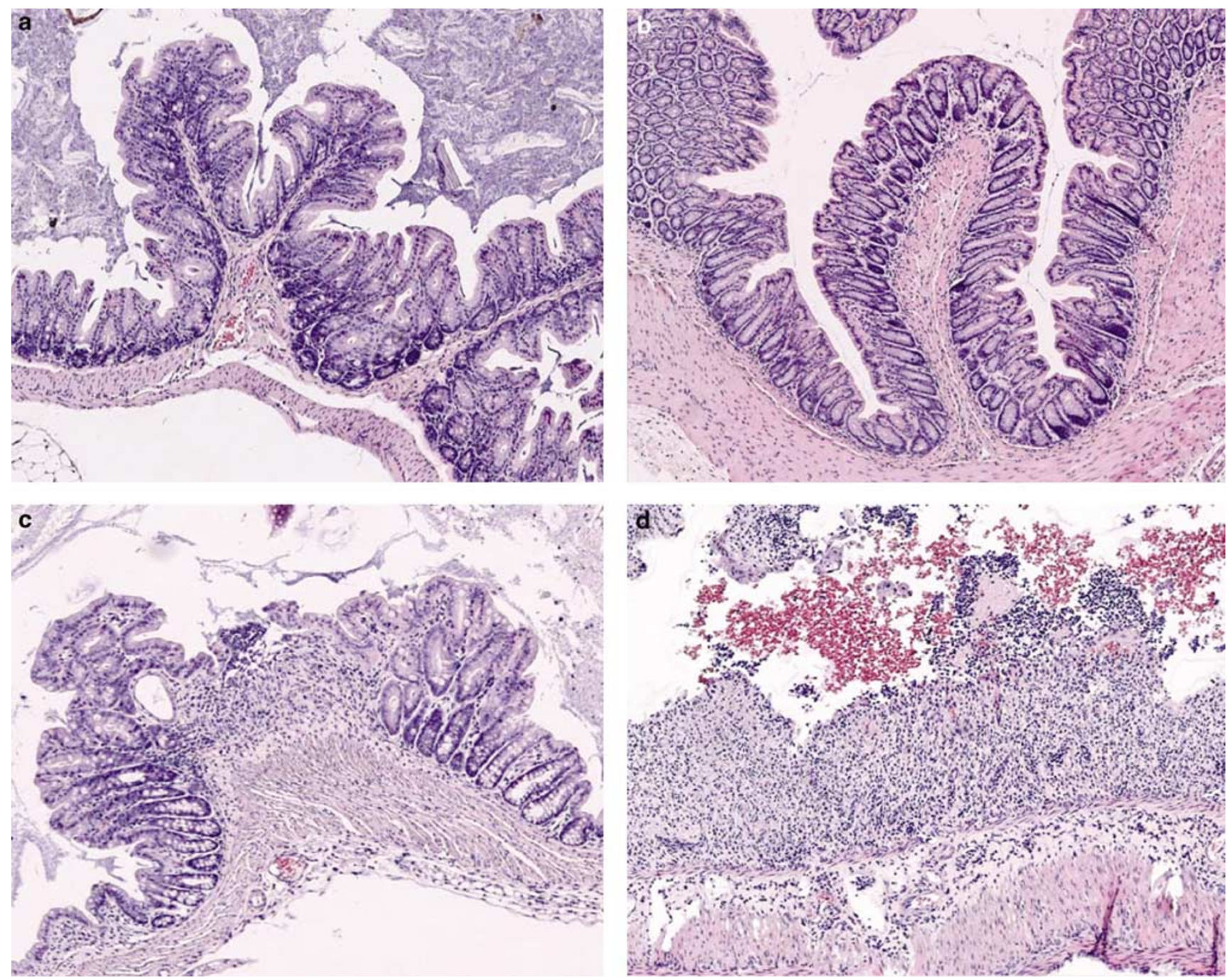

Figure 3 DSS-induced murine colitis is enhanced by CD98 activation. Histological sections of mouse colon from control mouse treated i.p. with isotype $(0.5 \mathrm{mg} / \mathrm{kg}$ ) (a), experimental mouse treated i.p. with $0.5 \mathrm{mg} / \mathrm{kg}$ mouse anti-CD98 (b), experimental mouse treated with $3 \%$ DSS alone (c), or experimental mouse treated with $3 \%$ DSS and $0.5 \mathrm{mg} / \mathrm{kg}$ mouse anti-CD98 antibody (d). Magnification $\times 40$. The results presented are representative of six different experiments.

loss when compared to that in mice treated with DSS alone (Figure 4). In contrast to anti-CD98 treatment, isotype control antibody treatment did not change the severity of DSS colitis (Histological activity score: 3). Finally, we examined whether DSS treatment increased the expression of CD98 in the colonic tissues of DSS-treated mice. As shown in Figure 4b, CD98 expression was increased as early as day 2 after DSS treatment. In contrast, CD98 protein expression in IFN $\gamma-/-$ mice treated with DSS was not affected (Figure 4c), suggesting that the DSS-mediated induction of CD98 protein expression is IFN $\gamma$-mediated.

\section{Anti-CD98 Does Not Affect Caco2-BBE Monolayer Reconstitution (Wound-Healing)}

Caco2-BBE cells were grown to confluency on electrodes coated with anti-mouse CD98 or the goat isotype control. Resistance was measured and found to be about $40000 \Omega$ in both cases; Caco2-BBE monolayers on electrodes coated with anti-mouse CD98 had resistances of $46000 \pm 5000 \Omega(n=3)$, whereas Caco2-BBE monolayers grown on the goat isotype had resistances of $38500 \pm 7000 \Omega(n=4)$. As shown in Figure 5a, the application of the high field pulse led to a drastic drop in the resistance of monolayers on both anti-CD98 (Figure 5a, red trace: $8000 \pm 3000 \Omega ; n=3$ ) and the goat isotype (Figure 5a, green trace; $7200 \pm 1200 \Omega ; n=4$ ), indicating that the wound model was successfully applied. The time courses of wound-healing were similar for Caco2BBE monolayers grown on anti-mouse CD98 and the goat isotype; the monolayers recovered within $10 \mathrm{~h}$ and displayed resistances even slightly superior to their prewounding resistances (Figure 5a). Unwounded Caco2-BBE monolayers grown on anti-mouse CD98 (red trace) or goat isotype (green 

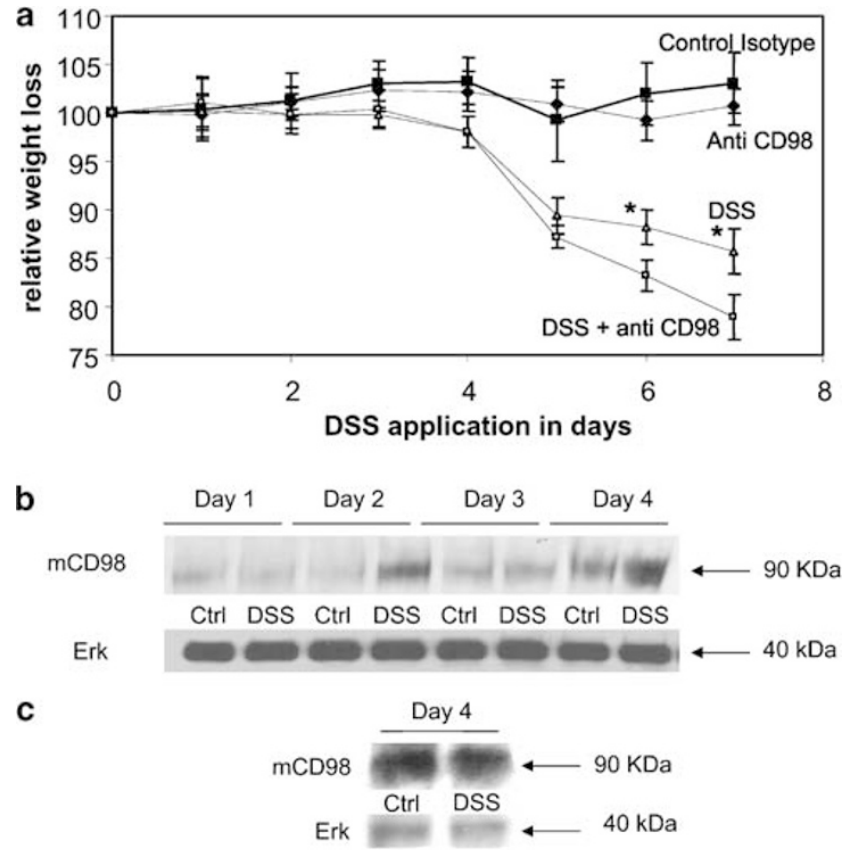

Figure 4 Activation of CD98 in DSS-induced murine colitis enhanced weight loss. (a) Body weight was monitored daily and data are expressed \pm s.e.m. with $n=6$ mice per condition. Statistically significant differences are noted as ${ }^{*} P<0.05$ and ${ }^{*} P<0.001$. The data presented are representative of three different experiments showing similar results. (b) Western blot analysis of CD98 protein in mouse intestine after DSS treatment. Adult mice were killed at days 1 (D1), 2 (D2) 3 (D3), and 4 (D4) after DSS treatment. Colonic segments were harvested and tested for CD98 protein content. Total proteins $(50 \mu \mathrm{g})$ were subjected to $7.5 \%$ SDS-polyacrylamide gel electrophoresis followed by transfer to a nitrocellulose membrane. The blot was immunostained with a goat anti-mouse CD98 antibody, and then stripped and re probed with anti-ERK to demonstrate equivalent loading. Ctrl, untreated; DSS, DSS-treated. Blots presented are representative of six different experiments. (c) CD98 protein expression in IFN $\gamma-/-$ mice without DSS treatment (CTRL) or treated with DSS (DSS) for 4 days, assayed as in (b). Blots presented are representative of three different experiments.

trace) showed stable resistances during the experimental time course (Figure 5a). These experimental results were confirmed by microscopy of wounded and control monolayers grown on electrodes coated with anti-mouse CD98 or the goat isotype (Figure 5b).

\section{Anti-CD98 Inhibits Wound Healing in Caco2-BBE Cell Monolayers Pretreated with DSS}

Confluent Caco2-BBE monolayers were grown on electrodes precoated with anti-mouse CD98 antibody or with goat isotype, and then treated with $3 \%$ DSS for $20 \mathrm{~h}$. As shown in Figure 5c, DSS treatment did not affect the resistances of Caco2-BBE monolayers grown on electrodes precoated with antimouse CD98 $(R=55000 \pm 7000 \Omega ; n=4)$ or the goat isotype $(R=65000 \pm 8200 \Omega ; n=4)$. Application of the high field pulse led to a drastic drop of resistance in Caco2-BBE monolayers grown on both anti-mouse CD98 (Figure 5c, dark cyan trace; $R=5000 \pm 1100 \Omega ; n=4)$ and the goat isotype (Figure 5b, pink trace; $R=5100 \pm 900 \Omega ; n=4$ ). Interestingly, DSS-treated Caco2-BBE cells grown on electrodes precoated with anti-mouse CD98 did not reach confluence by $20 \mathrm{~h}$ postwounding (Figure 5c, dark cyan trace), while those grown on the goat isotype did achieve confluence within this time (Figure 5c, pink trace). However, the DSS-treated Caco2-BBE monolayers grown on the isotype control healed more slowly ( $\sim 15 \mathrm{~h}$; Figure 5a) than comparable cells untreated with DSS ( $\sim 10 \mathrm{~h}$; Figure 5a). Caco2-BBE monolayers grown on anti-mouse CD98 (Figure 5a, red trace) or the goat isotype (Figure 5a, green trace) and treated with DSS but not wounded displayed a slight decrease in resistance during the time of the experiment. We further confirmed our observations by microscopic examination of Caco2BBE monolayers $20 \mathrm{~h}$ postwounding, which showed the confluence of the monolayer grown on the goat isotype (Figure 5d, DSS with wound) and the incomplete healing of the monolayer grown on anti-mouse CD98 (Figure 5d, DSS + anti-CD98 with wound).

\section{Discussion}

The role played by CD98 in colonic epithelial cells is poorly understood. The natural activators of CD98 remain unclear, although galectin-3 has been proposed as one candidate. ${ }^{25}$ Many of the previous CD98 functional studies have used anti-CD98 antibodies as agonists, an approach that has proven useful for the study of signaling in a great many. ${ }^{26}$ Indeed, our group has shown that CD98 is involved in signal recognition and transduction in Caco2-BBE cells, ${ }^{9,12}$ these signaling events may be mediated through $\beta 1$ integrins $/ \mathrm{Ca}^{2+}$, as CD98 is known to interact with integrins such as $\beta 1$ integrins and ICAM-1. ${ }^{12}$ In the present study, we demonstrate that the CD98 glycoprotein is expressed in Caco2-BBE monolayers, in the human intestine, and in the mouse small and large intestinal mucosa, and sought to better define the function of CD98 in these tissues.

It has been suggested that the increased epithelial expression of cell surface proteins in patients suffering from IBD occurs through a number of different pathways, including the production of cytokines by activated mucosal lymphocytes. ${ }^{27}$ IFN $\gamma$ is present at high levels in IBD tissues, where it prepares enterocytes to function in host defense. In addition, inhibition of endogenous IFN $\gamma$ by antibodies has been shown to ameliorate the chronic stage of colitis, indicating that IFN $\gamma$ is likely a key mediator for intestinal inflammation. ${ }^{28}$ Here, we demonstrated that IFN $\gamma$ increases CD98 glycoprotein expression in Caco2-BBE monolayers. This observation is consistent with the CD98 upregula- 

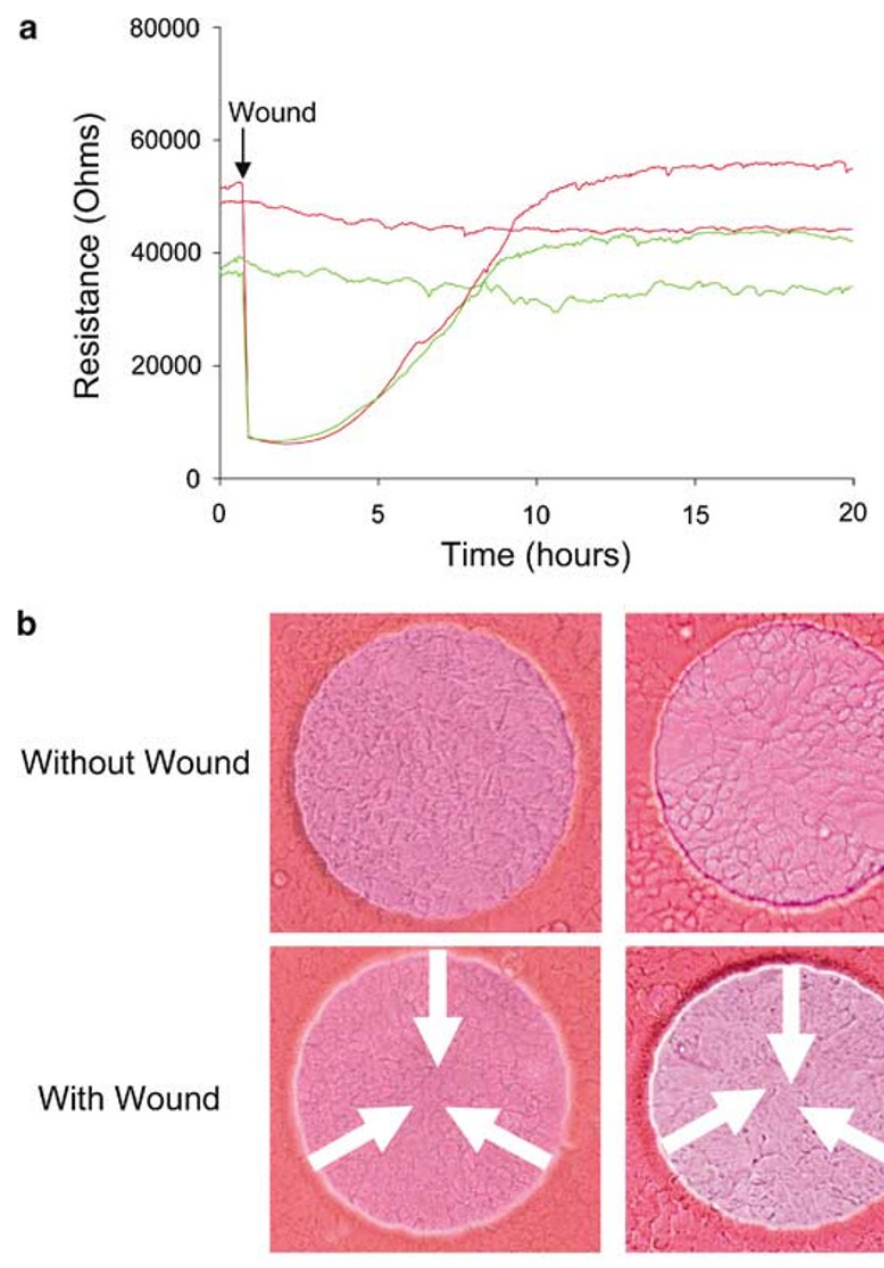

ctrl
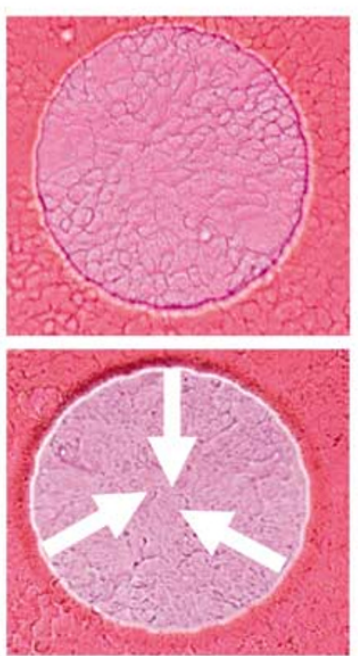

Anti-CD98

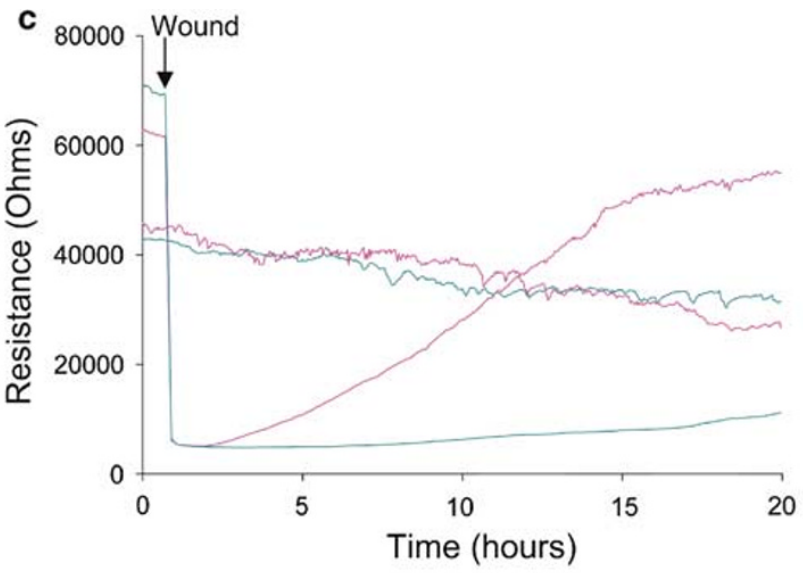

Figure 5 Anti-CD98 does not affect Caco2-BBE monolayer wound-healing. (a) Confluent Caco2-BBE cells were seeded in ECIS plates containing electrodes precoated with goat anti-mouse CD98 (red traces) or control isotype (green traces). At confluency, monolayers were submitted (wounded) or not (ctrl) to an elevated voltage pulse of $40 \mathrm{kHz}$ frequency, $4.5 \mathrm{~V}$ amplitude, and $30 \mathrm{~s}$ duration. Cells surrounding the wound were then allowed to reconstitute the monolayer. (b) At the end of the experiments, cells were washed twice in PBS and then photographed. Arrows indicate the direction of cell repair and migration. (c) Anti-CD98 inhibits wound healing in Caco2-BBE cell monolayers pretreated with DSS and grown on goat anti-mouse CD98 (red traces) or control isotype (green traces). At confluency, monolayers were submitted (wounded) or not (ctrl) to an elevated voltage pulse of $40 \mathrm{kHz}$ frequency, $4.5 \mathrm{~V}$ amplitude, and $30 \mathrm{~s}$ duration. Cells surrounding the wound were then allowed to reconstitute the monolayer. (d) At the end of the experiments, cells were washed twice in PBS and photographed. Arrows indicate the direction of cell repair and migration. Each trace represents the average of at least three wells.

tion found on colonic epithelial cells isolated from inflamed IBD tissue. ${ }^{13}$ These findings suggest that CD98 is up-regulated in inflamed epithelial cells, and that activation of CD98 might be involved in signal recognition and transduction steps, such as protein phosphorylation (eg FAK phosphorylation) and/or $\mathrm{Ca}^{2+}$ signaling events. ${ }^{29}$

To evaluate a possible inflammatory role for CD98 in intestinal epithelial cells, we injected a mouse anti-CD98 mAb into mice, where it should bind to CD98 proteins expressed in intestinal epithelial cells. Since CD98 is not expressed on freshly isolated, resting peripheral blood mononuclear cells, the anti-CD98 antibody was not expected to activate lymphocytes. ${ }^{13,14}$ Under our experimental conditions, the anti-CD98 antibody did not induce macroscopic intestinal inflammation in vivo. One important aspect of IBD is that immune cells can diffusely infiltrate the intestine without triggering obvious morphological, clinical, and endoscopic evidence of inflammation, ${ }^{15}$ suggesting that it may be formally possible that CD98 affects some intestinal epithelial cell function(s) without inducing morphological changes. However, the transepithelial resistance of the Caco2-BBE monolayers was not affected by activation of epithelial CD98, indicating that CD98 may not trigger an inflammatory response. These observations are not surprising since intestinal inflammations such as IBD are known to result from multiple factors. 
Based on the above observations, we speculated that the establishment of an inflammatory context might be necessary for anti-CD98 to have an effect on the intestine. We chose DSS-induced colitis as a model intestinal inflammation, because this model has the benefit of simplicity and highly uniform lesions. ${ }^{15}$ Furthermore, DSS-induced colitis is viewed as a T-cell-independent model because it is observed in T-cell-deficient animals such as SCID mice. ${ }^{15}$ In contrast, markers of lymphocyte activation, including the $4 \mathrm{~F} 2$ antigen, the T9 transferrin receptor (CD71), and the IL-2 receptor, are increased among isolated intestinal $\mathrm{B}$ cells and $\mathrm{T}$ cells in intestinal inflammation induced by $\mathrm{DSS},{ }^{28}$ indicating that DSS-induced colitis is a useful model for examining the role of CD98 in the colonic mucosa and in T cells.

Oral administration of DSS for 7 days induced acute colitis in mice. Histologically, the colons were characterized by multifocal dropouts of entire crypts in all parts of the colon and cecum, as compared to the normal colons. Crypt abscesses were observed in some animals. The inflammation was mostly confined to the mucosa, with extensive edema of the submucosa in some cases. When anti-CD98 was administered in combination with DSS, histological studies revealed enhanced infiltration of inflammatory cells, especially neutrophils, and mucosal cell disruption in DSS/anti-CD98-treated mice compared to mice treated with DSS alone.

Interestingly, addition of DSS did not significantly affect the integrity of Caco2-BBE monolayers, suggesting that DSS requires additional inflammatory mediators to trigger intestinal inflammation. However, wounded Caco2-BBE monolayers pretreated with DSS showed slower recoveries of their epithelial barrier functions, perhaps indicating that increased cellular exposure to luminal agents might contribute to persistent colitis in vivo. Thus, active inflammation persisting within the colonic mucosa and disturbances in mucosal healing may both contribute to the intractability of ulcerative colitis.

It is known that $\mathrm{TNF} \alpha$ and IFN $\gamma$ are involved in perpetuation of chronic DSS-induced colitis. ${ }^{15} \mathrm{We}$ found that CD98 expression was increased in DSStreated mice, at least partially mediated by IFN $\gamma$. Consistent with this, our in vitro results showed that epithelial expression of CD98 was enhanced in IFN $\gamma$-treated Caco2-BBE cells. DSS is believed to cause colitis by interfering with intestinal barrier function and/or stimulating local inflammation within the colon via up-regulation of both Th1 and Th2 cytokines and inflammatory mediators. ${ }^{28}$ This may explain the increased expression of CD98 in intestinal epithelial cells during inflammation.

We have previously speculated that the specific molecular ratio between the CD98/amino-acid transporter heterodimer and $\beta 1$ integrins might determine the polarity of epithelial cells. During inflammation, the overexpression of CD98 in intestinal epithelial cells will change this specific ratio. One of the potential consequences will be a loss of epithelial cell polarity, which is known to be a characteristic feature of IBD.

In sum, our data collectively indicate that (i) CD98 is upregulated during inflammation and (ii) activation of CD98 protein aggravates intestinal inflammation. We propose that during inflammation in vivo, CD98 is up-regulated and that natural CD98-binding proteins are released. The combination of these events may play an important role in the induction and perpetuation of intestinal inflammation by decreasing cell migration.

\section{Acknowledgement}

This work was supported by the National Institute of Diabetes and Digestive and Kidney Diseases under a center grant (R24-DK-064399) and R01-DK061941-01A1 (to D Merlin), R01DK064711-01 (to SV Sitaraman), and a Research Fellowship Award from the Crohn's and Colitis Foundation of America (to L Charrier). T Kucharzik was supported by the Deutsche Forschungsgemeinschaft (DFG) (KU 1328/1-1).

\section{References}

1 Sandborn WJ, Yednock TA. Novel approaches to treating inflammatory bowel disease: targeting alpha4 integrin. Am J Gastroenterol 2003;8:2372-2382.

2 Musch MW, Clarke LL, Mamah $\mathrm{D}$, et al. $\mathrm{T}$ cell activation causes diarrhea by increasing intestinal permeability and inhibiting epithelial $\mathrm{Na}^{+} / \mathrm{K}^{+}$-ATPase. J Clin Invest 2002;10:1739-1747.

3 Merlin D, Si-Tahar M, Sitaraman SV, et al. Colonic epithelial hPepT1 expression occurs in inflammatory bowel disease: transport of bacterial peptides influences expression of MHC class 1 molecules. Gastroenterology 2001;20:1666-1679.

4 Mastroberardino L, Spindler B, Pfeiffer R, et al. Aminoacid transport by heterodimers of 4F2hc/CD98 and members of a permease family. Nature 1998;395:288-291.

5 Verrey F. System L. Heteromeric exchangers of large, neutral amino acids involved in directional transport. Pflugers Arch 2003;445:529-533.

6 Haynes BF, Hemler ME, Mann DL, et al. Characterization of a monoclonal antibody (4F2) that binds to human monocytes and to a subset of activated lymphocytes. J Immunol 1981;126:1409-1414.

7 Nakamura E, Sato M, Yang H, et al. 4F2 (CD98) heavy chain is associated covalently with an amino acid transporter and controls intracellular trafficking and membrane topology of 4F2 heterodimer. J Biol Chem 1999;274:3009-3016.

8 Rossier G, Meier C, Bauch C, et al. LAT2, a new basolateral 4F2hc/CD98-associated amino acid transporter of kidney and intestine. J Biol Chem 1999; 274:34948-34954.

9 Merlin D, Sitaraman S, Liu X, et al. CD98-mediated links between amino acid transport and beta 1 integrin distribution in polarized columnar epithelia. J Biol Chem 2001;276:39282-39289. 
10 Bauch C, Forster N, Loffing-Cueni D, et al. Functional cooperation of epithelial heteromeric amino acid transporters expressed in madin-darby canine kidney cells. J Biol Chem 2003;278:1316-1322.

11 Zent R, Fenczik CA, Calderwood DA, et al. Classand splice variant-specific association of CD98 with integrin beta cytoplasmic domains. J Biol Chem 2000; 275:5059-5064.

12 Liu X, Charrier L, Gewirtz A, et al. CD98 and intracellular adhesion molecule I regulate the activity of amino acid transporter LAT-2 in polarized intestinal epithelia. J Biol Chem 2003;278:23672-23677.

13 Fais S, Pallone F. Ability of human colonic epithelium to express the $4 \mathrm{~F} 2$ antigen, the common acute lymphoblastic leukemia antigen, and the transferrin receptor. Studies in inflammatory bowel disease and after in vitro exposure to different stimuli. Gastroenterology 1989;97:1435-1441.

14 Fais S, Capobianchi MR, Silvestri M, et al. Interferon expression in Crohn's disease patients: increased interferon-gamma and -alpha mRNA in the intestinal lamina propria mononuclear cells. J. Interferon Res 1994;14:235-238.

15 Fiocchi C. Inflammatory bowel disease: etiology and pathogenesis. Gastroenterology 1998;115:182-205.

16 Peterson MD, Mooseker MS. An in vitro model for the analysis of intestinal brush border assembly. I. Ultrastructural analysis of cell contact-induced brush border assembly in Caco-2BBe cells. J Cell Sci 1993; 105:445-460.

17 Buyse M, Sitaraman SV, Liu X, et al. Luminal leptin enhances CD147/MCT-1-mediated uptake of butyrate in the human intestinal cell lineCaco2-BBE. J Biol Chem 2002;277:28182-28190.

18 Liu X, Charrier L, Gewirtz A, et al. CD98 and intracellular adhesion molecule I regulate the activity of amino acid transporter LAT-2 in polarized intestinal epithelia. 2003;278:23672-23677.

19 Buyse M, Charrier L, Sitaraman S, et al. Interferongamma increases hPepT1-mediated uptake of di-tripeptides including the bacterial tripeptide fMLP in polarized intestinal epithelia. Am J Pathol 2003;163: 1969-1977.

20 Grynkiewicz G, Poenie M, Tsien RY. A new generation of $\mathrm{Ca}^{2+}$ indicators with greatly improved fluorescence properties. J Biol Chem 1985;260:3440-3450.

21 Rintoul RC, Buttery RC, Mackinnon AC, et al. Crosslinking CD98 promotes integrin-like signaling and anchorage-independent growth. Mol Biol Cell 2002; 13:2841-2852.

22 Tsukada Y, Nakamura T, Iimura M, et al. Cytokine profile in colonic mucosa of ulcerative colitis correlates with disease activity and response to granulocytapheresis. Am J Gastroenterol 2002;97: 2820-2828.

23 Bouma G, Strober W. The immunological and genetic basis of inflammatory bowel disease. Nat Rev Immunol 2003;3:521-533.

24 Schreiber S, MacDermott RP, Raedler A, et al. Increased activation of isolated intestinal lamina propria mononuclear cells in inflammatory bowel disease. Gastroenterology 1991;101:1020-1030.

25 Dieleman LA, Ridwan BU, Tennyson GS, et al. Dextran sulfate sodium-induced colitis occurs in severe combined immunodeficient mice. Gastroenterology 1994; 107:1643-1652.

26 Axelsson LG, Landstrom E, Goldschmidt TJ, et al. Dextran sulfate sodium (DSS) induced experimental colitis in immunodeficient mice: effects in CD4(+) -cell depleted, athymic and NK-cell depleted SCID mice. Inflamm Res 1996;45:181-191.

27 Yacyshyn BR. Activated CD19+ B cell lamina propria lymphocytes in ulcerative colitis. Immunol Cell Biol 1993;71:265-274.

28 Dieleman LA, Palmen MJ, Akol H, et al. Chronic experimental colitis induced by dextran sulphate sodium (DSS) is characterized by Th1 and Th2 cytokines. Clin Exp Immunol 1998;114:385-391.

29 Dong S, Hughes RC. Galectin-3 stimulates uptake of extracellular $\mathrm{Ca}^{2+}$ in human Jurkat T-cells. FEBS Lett 1996;395:165-169. 